\title{
Enhanced magneto-optical effect due to interface alloy formation in Co-Pt (llll 111$)$ ultrathin films upon thermal annealing
}

\author{
M.-T. Lin ${ }^{\mathrm{a}}{ }^{*}$, C.C. Kuo ${ }^{\mathrm{a}}$, J.W. Ho ${ }^{\mathrm{a}}$, Y.E. Wu ${ }^{\mathrm{b}}$, H.Y. Her ${ }^{\mathrm{b}}$, C.S. Shern ${ }^{\mathrm{b}}$, H.L. Huang ${ }^{\mathrm{a}}$ \\ ${ }^{a}$ Department of Physics, National Taiwan University, 106 Taipei, Taiwan \\ ${ }^{\mathrm{b}}$ Department of Physics, National Taiwan Normal University, 117 Taipei, Taiwan
}

Received 2 August 1999; accepted 22 October 1999

\begin{abstract}
After postdeposition annealing in the temperature range of 500-800 K, a highly enhanced magneto-optical Kerr response up to a maximal value $300 \%$ of that before annealing, is observed for 1-2 ML ultrathin Co-Pt (1 111 ) films. With help of low energy electron diffraction and Auger electron spectroscopy, this enhancement of magneto-optical response is found to be correlated to the Co-Pt alloy formation at interface. (C) 2001 Elsevier Science B.V. All rights reserved.
\end{abstract}

Keywords: Interface alloy; Magneto-optical Kerr effect; Perpendicular magnetic anisotropy; Ultrathin film; Thermal annealing

\section{Introduction}

Co-Pt based systems, such as multilayers and alloy thin films, have attracted intensive interest due to their application in data storage technology as promising magneto-optical media [1-3]. Numerous reports on this system indicate a complicated interplay between the magnetic or magneto-optical behavior and the $\mathrm{Co}-$ $\mathrm{Pt}$ alloy composition as well as the crystalline structure, which are sensitively affected by the growth temperature and annealing temperature [3-6]. The physical origin and a complete understanding of many findings in these studies often require a detailed characterization of crystalline structure and chemical composition at the interface of multilayers or thin films. As compared to multilayers, ultrathin films provides an ideal system for a controllable Co-Pt

\footnotetext{
Corresponding author. Fax: +886-2-2363-9984. E-mail address: mtlin@phys.ntu.edu.tw (M.-T. Lin).
}

interface. This is because, in application of many surface sensitive analytical techniques, the structural and composition evolution at interface can be definitely extracted, and the related effects on magnetism can be thus more clearly identified. In this work, we will focus on evolution of magnetic and magnetooptical properties of the Co-Pt $\left(\begin{array}{lll}1 & 1 & 1\end{array}\right)$ films in an ultrathin limit upon postdeposition annealing. The annealing temperature is a sensitive parameter for the interdiffusion process at interface. Thermal annealing of the Co-Pt (lllll) films at appropriate temperatures can induce a metastable state of $\mathrm{Co}-\mathrm{Pt}$ alloy at interface, which gives rise to a large enhancement of magneto-optical effect.

\section{Experiment}

The experiments were carried out in an ultrahigh vacuum (UHV) chamber with base pressure in the range $5 \times 10^{-10}$ mbar. The UHV chamber was 
equipped with in situ facilities for low energy electron diffraction (LEED), Auger electron spectroscopy (AES), magneto-optical Kerr effect (MOKE), and thin film growth. A cobalt wire coil with purity of $99.997 \%$ was used to evaporate Co films. The pressure during the film growth was kept about $1 \times 10^{-9}$ mbar at an evaporation rate of about $0.1 \mathrm{ML} / \mathrm{min}$. The Co films were grown at $325 \mathrm{~K}$ and annealed afterwards at different temperatures up to $850 \mathrm{~K}$. The structural ordering and chemical composition of the sample were checked by LEED and AES, respectively. The magnetic and magneto-optical properties were monitored by the MOKE measurement, using a p-polarized He-Ne laser $(632.8 \mathrm{~nm})$ as the light source. The linear polarizer was put in front of the light detector for detecting the reflective signal in the s-wave direction only. The obtained reflective intensity change in such an arrangement is proportional to the Kerr rotation in the magneto-optical Kerr effect. The polar and longitudinal Kerr measurements were performed by simply rotating an electromagnet in the UHV chamber with a maximal magnetic of 950 Oe.

\section{Results and discussion}

According to our MOKE results, the Co-Pt $\left(\begin{array}{lll}1 & 1 & 1\end{array}\right)$ films grown at $325 \mathrm{~K}$ reveal a perpendicular anisotropy at coverages up to $5 \mathrm{ML}$, supplying a consistent and comparable system with the previous study [7]. Fig. 1 reveals the polar remanence Kerr signal as a function of the sample temperature for the 2.2

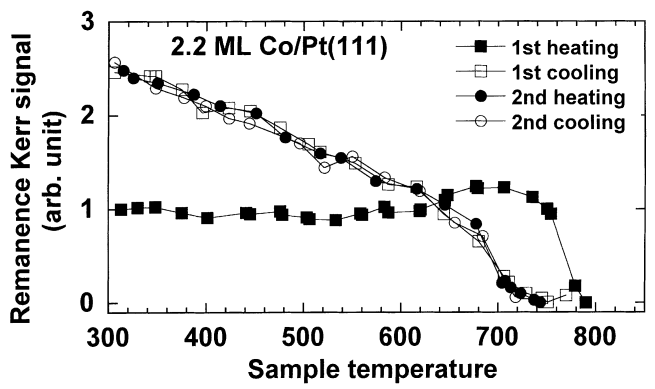

Fig. 1. Polar remanence Kerr signals as a function of the sample temperature for the 2.2 ML Co-Pt ( $\left.\begin{array}{lll}1 & 1 & 1\end{array}\right)$ for the first heating (solid squares), first cooling (empty squares), second heating (solid circles) and second cooling (empty circles) cycles.
ML Co-Pt (1 11 1). The Co film, as grown, is first heated from 325 to $790 \mathrm{~K}$. As indicated by the solid squares in Fig. 1, the polar remanence signal in the first heating procedure remains almost unchanged for the sample temperature up to $620 \mathrm{~K}$. Above $620 \mathrm{~K}$, the remanence Kerr signal, surprisingly, increases with the temperature and reaches a maximal value around $700 \mathrm{~K}$. For the temperature higher than $700 \mathrm{~K}$, the remanence Kerr signal drops quickly to zero at $790 \mathrm{~K}$. The finding of an increase in remanence signal with temperature deviates significantly from the behavior in usual ferromagnetic materials. After the first heating cycle, the sample is immediately cooled down to the room temperature. The corresponding remanence Kerr signal (empty squares in Fig. 1) is found to be not reversible with temperature, but increases with decreasing temperature and reaches at $325 \mathrm{~K}$, a value about $250 \%$ of that before heating. It is also clear that, between 640 and $790 \mathrm{~K}$, the remanence signal for the first cooling cycle is smaller than that for the first heating cycle. The sample is heated again to $790 \mathrm{~K}$ and then cooled down to the same temperature. Interestingly, the remanence Kerr signal is now reversible with temperature. The Kerr signals after the first heating cycle change reversibly with temperature. This finding indicates that, after the first

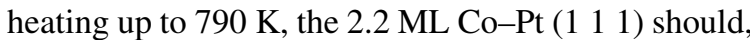
from the magnetic point of view, become another "magnetic system" with different intrinsic temperature dependence.

In order to further investigate the effect of the annealing temperature on the perpendicular anisotropy and magneto-optical effect, we anneal the 2.2 ML Co-Pt ( $\left.\begin{array}{lll}1 & 1 & 1\end{array}\right)$ at different temperatures for $3 \mathrm{~min}$. Fig. 2 compiles Kerr hysteresis loops measured at $325 \mathrm{~K}$ in the polar and longitudinal Kerr effect for the 2.2 ML Co-Pt (lllll 111 ) film annealed at different temperatures. The 2.2 ML film before annealing, as mentioned above, reveals a perpendicular anisotropy. After annealing at $710 \mathrm{~K}$, the perpendicular Kerr response at $325 \mathrm{~K}$ increases drastically about $250 \%$ as compared to the value before annealing. The $2.2 \mathrm{ML}$ films before annealing and after $710 \mathrm{~K}$ annealing still clearly reveal perpendicular anisotropy only. Annealing the film up to $850 \mathrm{~K}$, the perpendicular remanence Kerr signal increases further to an amazing value about $320 \%$ of that before annealing. Nevertheless, the coercivity for the polar 


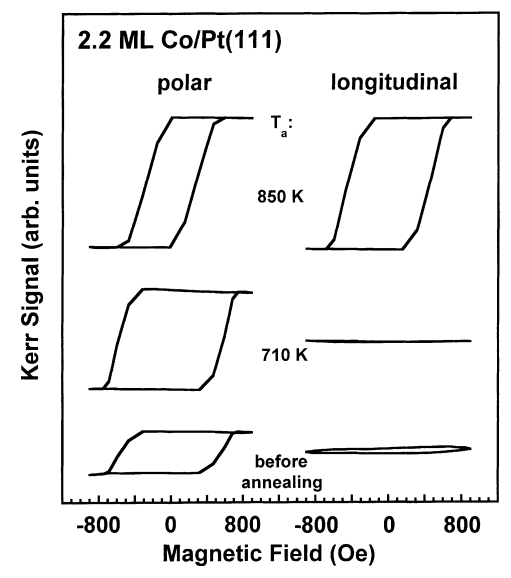

Fig. 2. Kerr hysteresis loops in the polar and longitudinal geometry for the $2.2 \mathrm{ML}$ Co-Pt $\left(\begin{array}{llll}1 & 1 & 1\end{array}\right)$ film annealed at different temperatures $T_{\mathrm{a}}$ for $3 \mathrm{~min}$. The Kerr signals were taken at $325 \mathrm{~K}$.

hysteresis loop become smaller and in the meantime, a significant in-plane signal is observed. This finding not only concerns the change in magneto-optical effects in polar or longitudinal geometry, but also clearly indicates a decrease of the perpendicular anisotropy and a raise of in-plane anisotropy or in-plane magnetic domain structure. This evolution of the perpendicular and in-plane anisotropy upon annealing at $850 \mathrm{~K}$ for the $2.2 \mathrm{ML}$ film may be understood if we take both processes into account, a complete diffusion of the Co into the Pt substrate and the decrease of perpendicular anisotropy due to the poor interface quality upon thermal annealing at an extremely high annealing temperature. Before most of Co atoms diffuse deeply into the substrate, the perpendicular Kerr signal, which is attributed to the interface anisotropy, decreases significantly due to the poor interface quality upon thermal annealing at $850 \mathrm{~K}$. Thus, before the Co atoms diffuses completely into the substrate and the ferromagnetic ordering of the total system vanishes, the in-plane Kerr signal or the in-plane magnetization appears.

For a deep insight into the origin of the enhancement of magneto-optical response, a more complete experiment including the MOKE, AES and LEED are performed for 1.0 ML Co-Pt ( $\left.\begin{array}{lll}1 & 1 & 1\end{array}\right)$. Fig. 3 combines the results of the remanence Kerr signal in both polar (squares) and longitudinal (circles) geometry, the

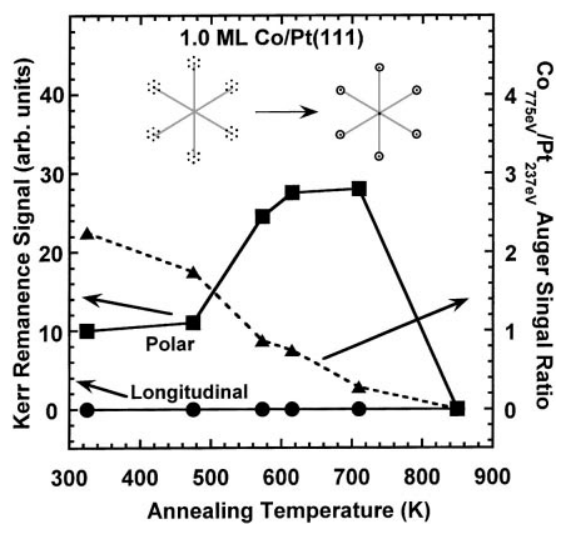

Fig. 3. Remanence Kerr signal in the polar (squares) and longitudinal (circles) geometry, the $\mathrm{Co}_{775 \mathrm{ev}} / \mathrm{Pt}_{237 \mathrm{eV}}$ Auger signal ratio (triangles) and the schematic LEED patterns for the 1.0 ML Co-Pt (llll 111$)$ annealed at different temperatures for $5 \mathrm{~min}$. The measurement temperature is $325 \mathrm{~K}$.

$\mathrm{Co}_{775 \mathrm{ev}} / \mathrm{Pt}_{237 \mathrm{ev}}$ Auger signal ratio (triangles) and the schematic LEED pattern for the 1.0 ML films annealed at different temperatures for $5 \mathrm{~min}$. All of the measurement temperatures are $325 \mathrm{~K}$. The longitudinal remanence Kerr signal vanishes for all annealing temperatures, indicating the absence of an in-plane ferromagnetic ordering. The polar remanence Kerr signal starts to increase drastically at the annealing temperature above $500 \mathrm{~K}$. At about $700 \mathrm{~K}$ annealing temperature, it reaches a maximal value about $300 \%$ of that before annealing. On the other hand, the $\mathrm{Co}-\mathrm{Pt}$ auger signal drops rapidly in the same annealing temperature range with the large enhancement of Kerr signal, indicating a strong Co-Pt interdiffusion at interface. Above the $700 \mathrm{~K}$ annealing temperature, the polar remanence Kerr signal drops to zero at $850 \mathrm{~K}$ annealing temperature. The fact that the $\mathrm{Co}-$ Pt Auger signal ratio goes down to zero for the $850 \mathrm{~K}$ annealing temperature indicates, as mentioned above for the 2.2 ML film, a complete diffusion of the magnetic Co into the $\mathrm{Pt}$ substrate. This explains why the ferromagnetic ordering disappears for this annealing temperature.

Furthermore, as indicated in Fig. 3, the annealing temperature can strongly affect the composition ratio of the Co and Pt. According to our further AES data (not shown here), a postdeposition annealing at appropriate annealing temperatures $(600 \mathrm{~K})$ can form a 
metastable phase with certain ratio of the $\mathrm{Co}$ and $\mathrm{Pt}$ composition, which is stable against a long time annealing and is accompanied with a constant magneto-optical optical Kerr effect.

Two characteristic LEED patterns, as shown in Fig. 3, are observed on the unannealed and annealed films. The first one reveals a six fold satellite fine structure around the $p(1 \times 1)$ main spots. This incommensurate LEED pattern is attributed to an incoherent epitaxy of the Co film on the Pt (lllll) substrate. Above the $550 \mathrm{~K}$ annealing temperature, which is almost the same as the onset annealing temperature of increase in polar remanence Kerr effect, the LEED pattern become $p(1 \times 1)$ with fuzzy spots. The inplane lattice constant is close to that of the $\mathrm{Pt}\left(\begin{array}{lll}1 & 1 & 1\end{array}\right)$ substrate. Together with the finding of a drop of Co-Pt Auger signal ratio, this structural transformation observed in the LEED pattern indicates a Co-Pt alloy formation at interface. The formation of Co-Pt alloy in this system has been reported in details in our previous study [8]. Corresponding to the structural evolution found in LEED upon thermal annealing, our previous data of ultraviolet photoelectron spectroscopy (UPS) also indicate a significant change in the valence electronic structure, which may be attributed to the formation of $\mathrm{Co}-\mathrm{Pt}$ bonds associated with a charge transfer between Co and Pt sites [8]. Based on the UPS data and the findings in Fig. 3, one can conclude that the enhancement of magneto-optical Kerr response is correlated with the formation of Co-Pt alloy at interface or surface.

In general, increase in magneto-optical Kerr response may be interpreted as increase in magnetization (this is also why we usually use MOKE to probe the magnetic properties) or enhancement of magnetooptical Kerr effect itself. However, the largely enhanced magneto-optical response, as shown above (up to about 300\%), seems unlikely to be due to change in magnetization mainly. Based on the previous experimental report on the composition dependence of the magnetic moment in Co-Pt alloy thin film system [10], the change of magneto-optical response due to increase in magnetization in our case is estimated to be only percent [11]. The correlation of the alloy formation at interface and the enhancement of the magneto-optical Kerr effect may be thus traced backed to the transfer of the strong spin-orbit coupling of the Pt into the magnetic Co orbital due to the hybridized electronic structure, as suggested in the theoretical study for the Co-Pt ultrathin multilayer system [9]. The significant enhancement of the magneto-optical Kerr signal in our case may be due to a special hybridization state between the $\mathrm{Co}$ and $\mathrm{Pt}$ through the thermal annealing, which is different from the expected structure in the Co-Pt alloy and multilayer systems as usual. This may explain the amazing enhancement factor (300\%) of magneto-optical Kerr signal found in our work, as compared to the previous study for the Co-Pt multilayer and Co-Pt alloy system [12].

\section{Conclusion}

Upon a thermal postdeposition annealing at appropriate temperatures, a giant enhancement of magnetooptical response up to about $300 \%$ is observed for the ultrathin Co-Pt ( $\left.\begin{array}{lll}1 & 1 & 1\end{array}\right)$ film in monolayer range. This finding is correlated to a Co-Pt alloy formation at interface, which results in a transfer of the strong spinorbit coupling of the $\mathrm{Pt}$ into the $\mathrm{Co}$, and in turn induces enhancement of the magneto-optical Kerr effect. Our results also suggest that the thermal annealing could be an effective postdeposition processing to modify the magneto-optical properties, and may become technologically relevant.

\section{Acknowledgements}

This work was supported by the National Science Council of Taiwan under Grant No. NSC 88-2112-M002-035 and NSC 87-2112-M-003-002.

\section{References}

[1] G.R. Harp, D. Weller, T.A. Rabedeau, R.F.C. Farrow, M.F. Toney, Phys. Rev. Lett. 71 (1993) 2493.

[2] T.C. Huang, R. Sayoy, R.F.C. Farrow, R.F. Marks, Appl. Phys. Lett. 62 (1993) 1353.

[3] C.J. Lin, G.L. Gorman, Appl. Phys. Lett. 61 (1992) 1600.

[4] P.W. Rooney, A.L. Shapiro, M.Q. Tran, F. Hellman, Phys. Rev. Lett. 75 (1995) 1843.

[5] P. He, W.A. McGahan, J.A. Wollam, F. Sequeda, T. McDaniel, H. Do, J. Appl. Phys. 69 (1991) 4021. 
[6] S. Shiomi, T. Nakakita, R. Tanaka, T. Kobayashi, M. Masuda, Jpn. J. Appl. Phys. 35 (1996) L213.

[7] J. Thiele, C. Boeglin, K. Hricovini, F. Chevrier, Phys. Rev. B 53 (1996) R11934.

[8] J.S. Tsay, C.S. Shern, Surf. Sci. 396 (1998) 313.

[9] G.Y. Guo, H. Ebert, J. Magn. Magn. Mater. 156 (1996) 173.
[10] T.R. McGuire, J.A. Aboaf, E. Klokholm, J. Appl. Phys. 55 (1984) 1951.

[11] M.-T. Lin, C.C. Kuo, H.Y. Her, Y.E. Wu, J.S. Tsay, C.S. Shern, J. Vac. Sci. Technol. A 17 (1999) 3045.

[12] D. Weller, H. Brandle, G. Gorman, C.-J. Lin, H. Notarys, Appl. Phys. Lett. 61 (1992) 2726. 